Article

\title{
In Vitro Properties and Virulence of Contemporary Recombinant Influenza B Viruses Harboring Mutations of Cross-Resistance to Neuraminidase Inhibitors
}

\author{
Clément Fage, Yacine Abed, Liva Checkmahomed, Marie-Christine Venable and Guy Boivin * \\ Research Center in Infectious Diseases of the CHUQ-CHUL and Laval University, 2705 Boulevard Laurier, \\ Québec City, QC G1V 4G2, Canada; Clement.Fage2@crchudequebec.ulaval.ca (C.F.); \\ yacine.abed@crchudequebec.ulaval.ca (Y.A.); liva.checkmahomed@crchudequebec.ulaval.ca (L.C.); \\ marie-christine.venable@crchudequebec.ulaval.ca (M.-C.V.) \\ * Correspondence: guy.boivin@crchudequebec.ulaval.ca; Tel.: +1-(418)-654-2705
}

Received: 30 November 2018; Accepted: 20 December 2018; Published: 22 December 2018

\begin{abstract}
Three neuraminidase inhibitors (NAIs: Oseltamivir, zanamivir and peramivir) are currently approved in many countries for the treatment of influenza A and B infections. The emergence of influenza B viruses (IBVs) containing mutations of cross-resistance to these NAIs constitutes a serious clinical threat. Herein, we used a reverse genetics system for the current B/Phuket/3073/2013 vaccine strain to investigate the impact on in vitro properties and virulence of H136N, R152K, D198E/N, I222T and N294S NA substitutions (N2 numbering), reported by the World Health Organization (WHO) as clinical markers of reduced or highly-reduced inhibition (RI/HRI) to multiple NAIs. Recombinant viruses were tested by NA inhibition assays. Their replicative capacity and virulence were evaluated in ST6Gall-MDCK cells and BALB/c mice, respectively. All NA mutants (excepted D198E/N) showed RI/HRI phenotypes against $\geq 2$ NAIs. These mutants grew to comparable titers of the recombinant wild-type (WT) IBV in vitro, and some of them (H136N, I222T and N294S mutants) induced more weight loss and mortality in BALB/c mice in comparison to the recombinant WT IBV. These results demonstrate that, in contemporary IBVs, some NA mutations may confer RI/HRI phenotypes to existing NAIs without altering the viral fitness. This reinforces the need for development of novel antiviral strategies with different mechanisms of action.
\end{abstract}

Keywords: influenza B; resistance; fitness; mouse model and neuraminidase mutation

\section{Introduction}

Influenza B viruses (IBVs) belong to the Orthomyxoviridae family. Contrasting with influenza A viruses (IAVs), IBVs cannot be subtyped based on their hemagglutinin (HA) and neuraminidase (NA) surface glycoproteins; nevertheless, antigenic and genetic characterizations allowed their separation into two lineages represented by B/Victoria/2/1987 and B/Yamagata/16/1988 strains [1]. Humans constitute the natural and predominant host for IBVs, although a few animal species, such as seals and domestic pigs, can also be infected [2].

Each year, IAV and IBV infections result in approximately one billion infections, causing an average of 3-5 million cases of severe diseases and up to 650,000 deaths worldwide [3]. For a long time, the contribution of IBVs to such seasonal influenza burden has been underestimated because these infections generally represented a small fraction of total cases and they were considered to cause milder infections in adults compared to IAVs [4]. Contrasting with this belief, a recent study demonstrated that influenza A and B infections induced similar lengths of hospital stay, intensive care 
unit admissions, and rates of death in hospitalized adults [5]. Accordingly, ferret studies demonstrated that IBVs replicate efficiently in the lower respiratory tract and cause infection with the same severity as IAVs [6]. Furthermore, the rate of seasonal IBV infections may surpass that of IAV cases in some influenza epidemics. For instance, during the 2017/2018 season, 63\% of all seasonal influenza cases reported in Europe were caused by IBVs [7].

The neuraminidase inhibitors (NAIs) constitute the main class of antivirals currently recommended against IAV and IBV infections. These compounds target the active site of the NA composed by 8 amino acids forming the catalytic site (R-118, D-151, R-152, R-224, E-276, R-292, R-371 and Y-406) and 11 framework residues (E-119, R-156, W-178, S-179, D-198, I-222, E-227, H274, E-277, N-294 and E-425) (N2 numbering herein and throughout the text). These residues are highly conserved among influenza A and B strains [8]. Currently, three NAIs (i.e., oseltamivir, zanamivir and peramivir) are widely available for the control of influenza infections [9]. Numerous in vitro studies and clinical reports involving IAVs and IBVs reported the emergence of strains with reduced susceptibility to NAIs, with and without being under drug pressure [10-12]. In most cases, IAV and/or IBV NAI-resistant variants contained amino acid substitutions within the active site of the NA enzyme [13]. Nevertheless, some mutations were also identified outside the active site involving mechanisms of resistance that remain to be clarified [14]. Noteworthily, while certain IBV NA mutations mediate a phenotype of reduced inhibition (RI; 5- to 50-fold increase in $\mathrm{IC}_{50}$ over the WT) or highly-reduced inhibition (HRI; $\geq 50$-fold increase) to a specific NAI without altering the susceptibility to other inhibitors, mutations associated with a cross-resistance phenotype to at least two NAIs have also been reported in various strains belonging to different genetic lineages [15]. The eventual emergence and dissemination of such variants represent a serious clinical concern.

In past studies, some variations were observed in the susceptibility phenotypes against NAIs when assessed in different IBV strains [10,12]. Moreover, little is understood about the impact of these NA changes on in vitro and in vivo viral fitness for current IBV strains. In this report, we seek to update our knowledge of NAI susceptibility phenotypes and in vitro/in vivo viral fitness of currently circulating IBV strains harboring NA mutations, conferring potential cross-resistance phenotypes to NAIs. For that purpose, we created a reverse genetics system using the contemporary influenza $\mathrm{B} /$ Phuket/3073/2013 genetic background, which allowed us to rescue and characterize IBV variants containing H136N, R152K, D198E/N, I222T and N294S NA substitutions reported by WHO as clinical molecular markers of RI/HRI to multiple NAIs [16].

\section{Materials and Methods}

\subsection{Virus and Cells}

The influenza B/Phuket/3073/2013 virus (Yamagata lineage-related vaccine strain since the 2015-2016 season) was obtained from NIBSC (code number \#17/248). Madin-Darby canine kidney (MDCK) and ST6GalI-MDCK cells (kindly provided by Y. Kawaoka from the University of Wisconsin, Madison, WI, USA) [17] were cultured in Minimum Essential Medium (MEM, Invitrogen, Carlsbad, CA, USA) supplemented with 10\% fetal bovine serum (FBS, Invitrogen), HEPES and antibiotics. The human embryonic kidney 293T cell line (ATCC, CRL-3216) was maintained in Dulbecco's modified Eagle's medium (DMEM, Invitrogen), supplemented with 10\% FBS and HEPES.

\subsection{Rescue of Recombinant IBVS}

To produce recombinant influenza B/Phuket/3073/2013 viruses, we used the bidirectional pBZ plasmid (kindly provided by B. Zhou from the University of New York, USA) [18]. Each viral segment was cloned into the $\mathrm{pBZ}$ plasmid. The pBZ-NA plasmid was used for inserting the selected NA mutations by PCR-mediated mutagenesis (Stratagene, La Jolla, CA, USA). Recombinant viruses were rescued as previously described [19], with minor modifications. Briefly, 293T/MDCK cells $\left(5 \times 10^{5}\right.$ cells of each line) were co-cultured in 6-well plates with DMEM $+10 \%$ FBS + HEPES for $48 \mathrm{~h}$ at $37^{\circ} \mathrm{C}$. Then, 
co-cultured cells were transfected with $1 \mu \mathrm{g}$ of each pBZ plasmid and lipofectamine $(3 \mu \mathrm{L} / \mu \mathrm{g}$ DNA $)$ in $1.2 \mathrm{~mL}$ of OptiMEM for $6 \mathrm{~h}$. The medium was replaced by DMEM $+0.1 \%$ bovine serum albumin (BSA) + bovine trypsin (TPCK-treated, Sigma, St-Louis, MO, USA) and co-cultures were incubated at $33^{\circ} \mathrm{C}$ for $72 \mathrm{~h}$ before harvesting viruses.

All IBV genomic segments within recombinant pBZ plasmids, as well as HA and NA genes of recombinant IBVs recovered during replicative capacity and mouse experiments, were sequenced using the ABI 3730 DNA Analyzer (Applied Biosystems, Carlsbad, CA, USA).

\subsection{Phenotype of Susceptibility to NAIs and Relative NA Activity}

NA inhibition assays using the methylumbelliferone N-acetylneuraminic acid (MUNANA, Sigma, St-Louis, MO, USA) substrate were performed for determining the median inhibition concentration $\left(\mathrm{IC}_{50}\right)$ for oseltamivir carboxylate (Hoffmann-La Roche, Basel, Switzerland), zanamivir (Sigma) and peramivir (BioCryst, Birmingham, AL, USA), as previously described [20]. The phenotypes of susceptibility to NAIs for recombinant IBVs were reported according to WHO criteria: NI, normal inhibition (<5-fold increase in $\mathrm{IC}_{50}$ over WT); RI, reduced inhibition (5- to 50-fold increase in $\mathrm{IC}_{50}$ over WT) and HRI, highly reduced inhibition (> 50-fold increase in $\mathrm{IC}_{50}$ over WT) [16].

To determine the impact of NA substitutions on the NA activity, recombinant NA proteins were expressed in HEK-293T cells, as previously described [20]. Briefly, confluent cells (in 12-well plates) were transfected with $500 \mathrm{ng}$ of the WT or mutant NA-pBZ plasmid + lipofectamine $(3 \mu \mathrm{L} / \mu \mathrm{g}$ DNA) and incubated for $6 \mathrm{~h}$ at $37^{\circ} \mathrm{C}$. The medium was completed with $500 \mu \mathrm{L}$ of DMEM $+20 \%$ FBS + HEPES, and transfected cells were incubated overnight at $37^{\circ} \mathrm{C}$. Then cells were harvested with $200 \mu \mathrm{L}$ of cold PBS $+0.02 \%$ EDTA, centrifuged $\left(1500 \times g, 5 \mathrm{~min}\right.$ at $\left.4{ }^{\circ} \mathrm{C}\right)$, washed with cold PBS and suspended in $200 \mu \mathrm{L}$ of $3.5 \mathrm{mM} \mathrm{CaCl}_{2}$ cold solution. NA activity assays were then performed as described above [20].

\subsection{In Vitro Viral Replication Kinetics}

The replicative capacities of recombinant IBVs were performed by infecting STG6GalI-MDCK cells in 12-well plates with 50 plaque forming units (PFUs)/well (M.O.I. of 0.0001 PFU/cell). Viruses were harvested at $24,48,72$ and $96 \mathrm{~h}$ post-infection (p.i.) and kept frozen at $-80{ }^{\circ} \mathrm{C}$ until titration by TCID 50 using STG6GalI-MDCK cells.

\subsection{Experimental Infection of Mice}

Groups of 6- to 8-week old female BALB/c mice (Charles River, Senneville, QC, Canada) $(n=10)$, were inoculated intranasally, under isoflurane anesthesia, with $5 \times 10^{6}$ PFUs of each recombinant IBV (this inoculum was previously determined to cause $\approx 100 \%$ of mortality in mice infected with the recombinant WT virus). A control group included 3 mice that were intranasally inoculated with saline. Mortality and weight loss were monitored daily for 10 days and the humane endpoint was determined at $20 \%$ of weight loss. On day 3 p.i., four mice per infected group were sacrificed and their lungs were collected and homogenized in $1 \mathrm{~mL}$ of PBS containing penicillin, streptomycin, and amphotericin B, using the Omni Tip homogenizer (OMNI International, Kennesaw, GA, USA). Cells were pelleted by centrifugation $\left(1500 \times g, 5 \mathrm{~min}, 4^{\circ} \mathrm{C}\right)$, and supernatants were used for determination of viral titers, expressed as TCID 50 per lung, using ST6GalI-MDCK cells.

All animal procedures were approved by the Institutional Animal Care Committee of Laval University, according to guidelines of the Canadian Council of Animal Care (permission number: 2015063-4).

\subsection{Statistics}

Replication kinetics, mouse weight loss, and lung viral titers were analyzed with one-way ANOVA Dunnett's multiple comparisons test between the WT and the mutant groups using GraphPad Prism 6.0 (GraphPad Software Inc., La Jolla, CA, USA). 


\section{Results}

\subsection{NAI Susceptibility Phenotypes of Recombinant B Viruses}

The recombinant B/Phuket/3073/2013 WT virus and its H136N, R152K, D198E/N, I222T and N294S variants were successfully rescued. In NA inhibition assays, all NA mutants except D198E/N variants demonstrated decreased viral susceptibilities to at least two NAIs among zanamivir, peramivir, and oseltamivir (Table 1). The R152K exhibited an HRI phenotype against the three NAIs, whereas a RI phenotype against these compounds was shared by the I222T and N294S mutants. The H136N variant with an HRI phenotype against zanamivir and peramivir remained susceptible to oseltamivir. Finally, the D198E/N mutants were susceptible to oseltamivir and zanamivir, although both had a RI phenotype to peramivir. In the absence of cross-resistance, the latter viruses were not further characterized.

The NA activities of recombinant H136N, D198E, D198N and I222T NA proteins were similar to that of the WT $(92 \%, 107 \%, 101 \%$ and 106\%, respectively) (Table 1), while the N294S NA protein showed a slight (but statistically significant) increase of activity (108\% vs the WT, $p<0.05)$. Finally, the R152K NA protein had a significantly reduced activity $(60 \%$ vs. the WT, $p<0.001)$ (Table 1$)$.

\subsection{Replicative Capacity of NAI-Resistant IBV Recombinants}

In replication kinetics experiments, the recombinant WT IBV and its H136N, I222T and N294S NA variants grew to comparable titers at different time-points (Figure 1). Viral titers ranged between $10^{3.2}$ and $10^{4.2} \mathrm{TCID}_{50}$ at $24 \mathrm{~h}$ to $10^{7.5}$ and $10^{8.2} \mathrm{TCID}_{50}$ at $72 \mathrm{~h}$. By contrast, the R152K mutant showed a significant reduction of the viral titer, compared to the WT $(p<0.01)$, that was only observed at the 72-h time point p.i. (Figure 1).

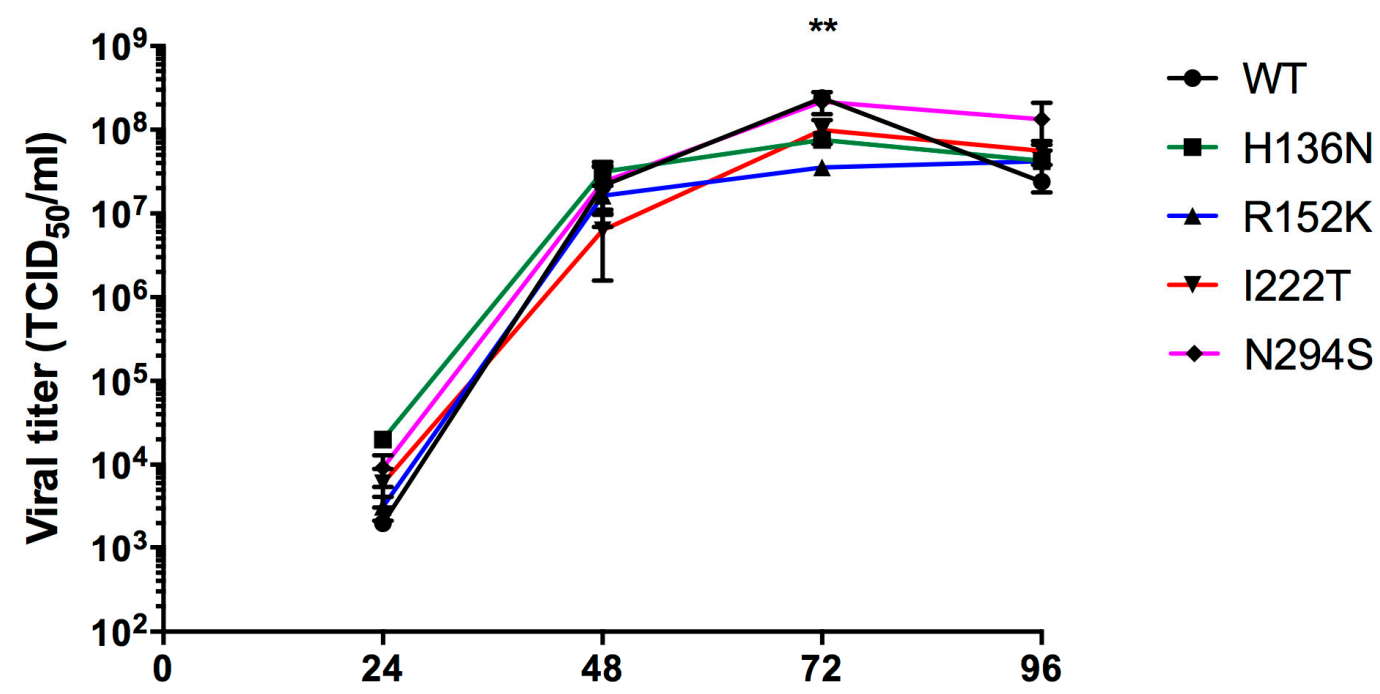

\section{Hours p.i.}

Figure 1. Replicative capacity of recombinant influenza B/Phuket/3073/2013 viruses in STG6GalI-MDCK cells. Cells were infected with a MOI $=0.0001$ in triplicate for each group and viruses were harvested at $24,48,72$ and $96 \mathrm{~h}$ p.i. and frozen at $-80^{\circ} \mathrm{C}$ before viral titration by $\mathrm{TCID}_{50}$. **, $p<0.01$ between WT and R152K groups using one-way ANOVA Dunnett's multiple comparisons of each mutant relative to the WT virus. 
Table 1. Phenotype of resistance to neuraminidase (NA) inhibitors for recombinant influenza B/Phuket/3073/2013 viruses.

\begin{tabular}{|c|c|c|c|c|c|c|c|c|c|c|c|c|c|c|c|c|c|}
\hline \multirow{3}{*}{$\frac{\text { Substitution }^{\mathrm{c}}}{\mathrm{WT}}$} & \multicolumn{14}{|c|}{ Neuraminidase Inhibitors $\mathrm{IC}_{50}$ values $(\mathrm{nM})^{a}$} & \multicolumn{3}{|c|}{ NA Activity ${ }^{b}(\%)$} \\
\hline & \multicolumn{2}{|c|}{ Oseltamivir } & \multirow{2}{*}{$\begin{array}{c}\text { Folds }^{\mathbf{d}} \\
1\end{array}$} & \multirow{2}{*}{ Phenotype $^{e}$} & \multicolumn{3}{|c|}{ Zanamivir } & \multirow{2}{*}{$\begin{array}{c}\text { Folds }^{\mathrm{d}} \\
1\end{array}$} & \multirow[t]{2}{*}{ Phenotype $^{\mathrm{e}}$} & \multicolumn{3}{|c|}{ Peramivir } & \multirow{2}{*}{$\begin{array}{c}\text { Folds }^{d} \\
1\end{array}$} & \multirow[t]{2}{*}{ Phenotype $^{e}$} & & & \\
\hline & 16.98 & \pm 5.11 & & & 0.76 & \pm & 0.12 & & & 0.74 & \pm & 0.13 & & & 100 & \pm & 1.87 \\
\hline $\mathrm{H} 136 \mathrm{~N}$ & 57.20 & \pm 11.41 & 3 & $\mathrm{NI}$ & 161.22 & \pm & 23.47 & 212 & HRI & 177.46 & \pm & 199.9 & 240 & HRI & 92 & \pm & 6.69 \\
\hline R152K & 1017.79 & \pm 108.71 & 60 & HRI & 63.83 & \pm & 2.79 & 84 & HRI & 422.63 & \pm & 45.98 & 572 & HRI & $60^{* * *}$ & \pm & 2.0 \\
\hline D198E & 75.89 & $\pm \quad 5.89$ & 4 & $\mathrm{NI}$ & 2.98 & \pm & 1.05 & 4 & NI & 15.53 & \pm & 0.90 & 21 & RI & 107 & \pm & 3.4 \\
\hline D198N & 52.30 & $\pm \quad 2.87$ & 3 & NI & 2.23 & \pm & 0.17 & 3 & NI & 6.92 & \pm & 0.36 & 9 & RI & 101 & \pm & 0.44 \\
\hline $\mathrm{I} 222 \mathrm{~T}$ & 111.25 & $\pm \quad 37.33$ & 6 & RI & 3.90 & \pm & 1.61 & 5 & RI & 19.48 & \pm & 5.91 & 26 & RI & 106 & \pm & 3.32 \\
\hline N294S & 178.85 & \pm 94.09 & 10 & RI & 13.97 & \pm & 8.25 & 18 & RI & 33.68 & \pm & 18.98 & 46 & RI & $108^{*}$ & \pm & 0.95 \\
\hline
\end{tabular}

a Means IC $_{50}$ values +/-SD were obtained from two independent experiments in duplicate; ${ }^{\mathrm{b}}$ NA activity was obtained from triplicate of a single experiment and results were standardized compared to the WT $(\%) ;{ }^{*}, p<0.05$ compared to the WT; ${ }^{* * *}, p<0.001$ compared to the WT; ${ }^{c}$ Substitutions are reported in N2 numbering; ${ }^{\mathrm{d}}$ Fold changes according to WT virus; e Resistance phenotype in accordance with WHO criteria: NI, Normal Inhibition ( $\leq 5$-fold increase for influenza B); RI, Reduced Inhibition (between 5- and 50-fold increase for influenza B); HRI, Highly Reduced Inhibition ( $>50$-fold increase for influenza B) 


\subsection{Virulence of Recombinant IBVs in Mice}

In experimental infections of BALB/c mice, the recombinant $\mathrm{H} 136 \mathrm{~N}, \mathrm{I} 222 \mathrm{~T}$ and N294S viruses induced body weight losses that were greater than that of the WT group. At day 4 p.i., we recorded a $14.96 \%$ body weight loss in the WT infected group while weight loss reached $22.38 \%(p<0.001), 19.9 \%$ $(p<0.05)$, and $21.37 \%(p<0.01)$ in the H136N-, I222T-, and N294S-infected groups, respectively. Finally, only $9.1 \%$ of all body weight loss was observed in mice infected with the recombinant R152K virus $(p<0.01)$ (Figure 2A).

A

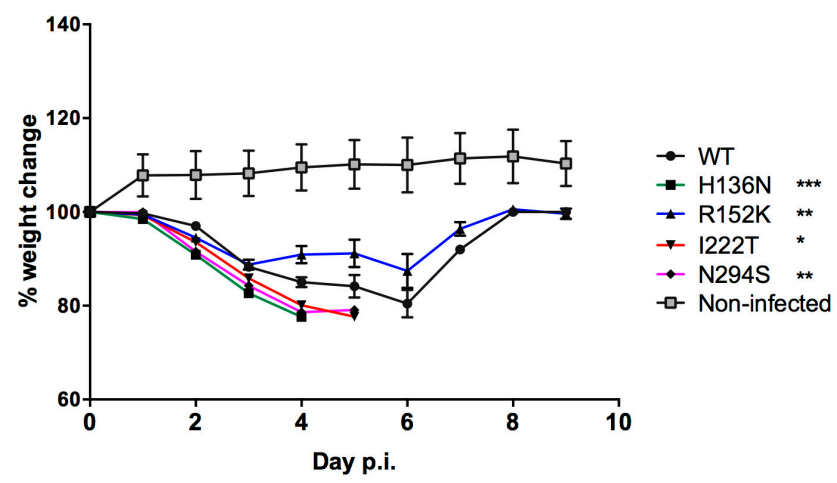

B
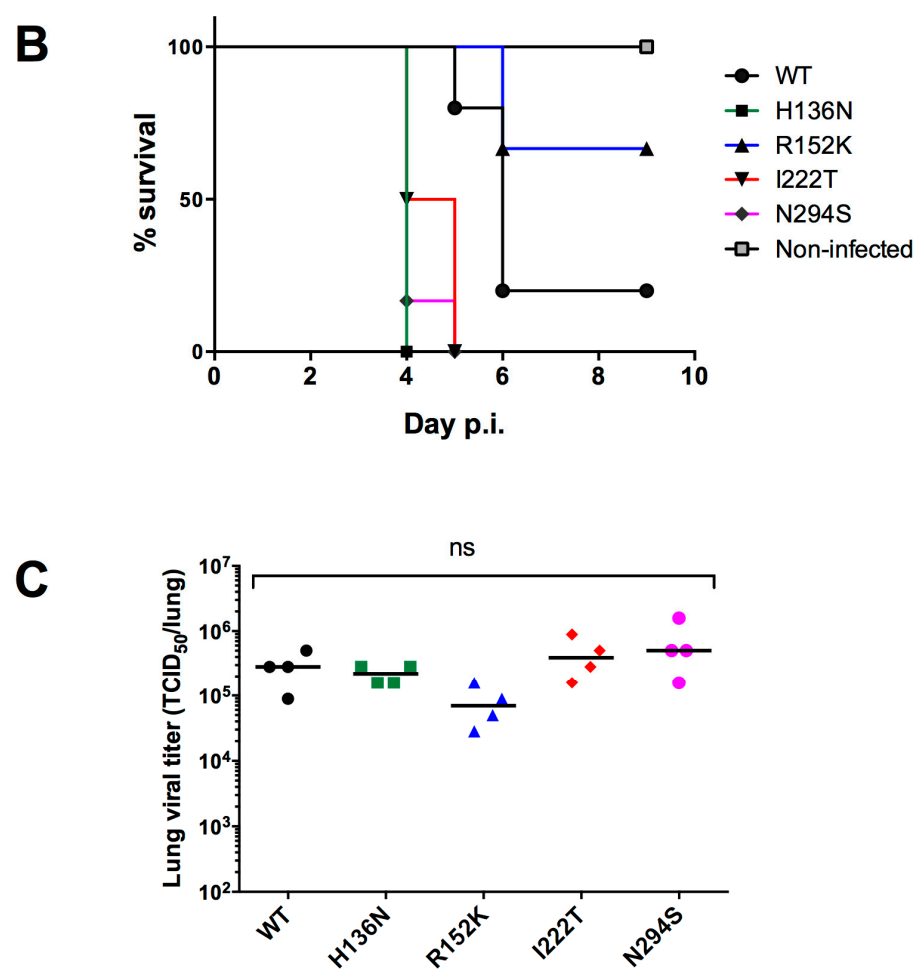

Figure 2. Virulence of recombinant IBVs with reduced susceptibility to NAIs. In each group, ten $\mathrm{BALB} / \mathrm{c}$ mice were infected by the intranasal route with $5 \times 10^{6} \mathrm{PFUs}$ of each recombinant B/Phuket/3073/2013 virus. Weight loss (A) and mortality (B) were monitored throughout the experiment. At day 3 p.i., 4 mice in each group were sacrificed and their lungs were harvested to determine lung viral titers (C). Statistical analyses of weight loss at day 4 (indicated in the legend) and lung titers were performed using one-way ANOVA, Dunnett's multiple comparisons test between the WT group and each of the mutant groups. ns, not significant, ${ }^{*}, p<0.05$ between WT and I222T, **, $p<0.01$ between WT and R152K or N294S groups, ${ }^{* * *}, p<0.001$ between WT and H136N groups. For the WT group, due to a technical problem during infection, one mouse was excluded from our analysis. 
All mice infected with the H136N, I222T and N294S recombinants died by day $4-5$ p.i., whereas mortality rates of $80 \%$ and $33 \%$ were observed in the WT and R152K groups, respectively (Figure 2B). Due to a technical problem during infection, one mouse infected with the WT virus was removed from our experiment analysis ( $n=5$ instead of 6$)$.

As shown in Figure 2C, high viral titers (between $10^{4.8}$ and $10^{5.7} \mathrm{TCID}_{50}$ per lung) were observed in the lungs of mice collected at day 3 p.i. Although a slightly reduced viral load was observed in the R152K infected group ( $10^{4.8} \mathrm{TCID}_{50}$ per lung), no significant difference was observed between the WT $\left(10^{5.4} \mathrm{TCID}_{50}\right.$ per lung) and each of the mutant groups $\left(10^{5.3}, 10^{5.6}\right.$ and $10^{5.7} \mathrm{TCID}_{50}$ per lung for $\mathrm{H} 136 \mathrm{~N}$, I222T, and N294S groups, respectively). Sequencing of viral HA and NA from lung homogenates confirmed the presence of the original NA mutation without other NA or HA changes.

\section{Discussion}

The eventual emergence and dissemination of multi-NAI resistant IBVs represent a serious clinical concern. Whether baloxavir marboxil, the new anti-influenza agent targeting the PA subunit of the viral polymerase [21], could constitute a suitable alternative against NAI-resistant IBV infections remains to be investigated. Indeed, rates of baloxavir resistance close to $20 \%$ were reported in H3N2-infected patients who received baloxavir therapy [22].

In this study, we used a unique contemporary viral background to investigate the impact of NA mutations previously associated with NAIs cross-resistance phenotypes in various clinical IBV strains. The use of the influenza B/Phuket/3073/2013 strain as a backbone in our experiments is important because this strain has been the vaccine component representing the Yamagata lineage in the northern hemisphere since the 2015-2016 season $[7,23,24]$. As part of this study, five NA substitutions (R152K, D198E, D198N, I222T and N294S) were selected on the basis of a recent WHO update on NAI resistance in clinical IAV and IBV isolates [16]. All these mutations were reported to confer a cross-resistance phenotype to at least two NAIs in IBV isolates from the Yamagata lineage. Influenza B/Memphis/20/1996-R152K and B/Rochester/02/2001-D198N variants were described in patients who received zanamivir and oseltamivir, respectively $[25,26]$. Additional R152K and D198N clinical isolates were identified in surveillance programs from untreated subjects $[12,15]$. The N294S substitution was observed in an immunocompromised child prior to oseltamivir therapy [27], and no additional cases have been reported since then. The D198E mutation detected in the B/Perth/211/2001 variant in the absence of NAI therapy also seemed to be rare in the Yamagata lineage, contrasting with the I222T substitution that was described in a recent surveillance update $[11,12]$. We also included the H136N substitution in our study since this variant was detected in a B/Laos/0406/2016 virus (Victoria lineage) with a cross-resistance phenotype [28].

Our NA inhibition assays data are in agreement with those of WHO regarding NAI susceptibilities and confirmed the RI/HRI phenotype to $\geq 2$ NAIs for all mutants, except for the D198E and D198N variants, that remained susceptible to oseltamivir (4- and 3-fold increase in $\mathrm{IC}_{50}$ values over WT, respectively) and zanamivir (4- and 3-fold increase, respectively) but had a RI phenotype against peramivir (21- and 9- fold increase, respectively). In previous studies, the D198E substitution conferred RI phenotypes to oseltamivir and zanamivir (in B/Perth/211/2001) or an RI phenotype against oseltamivir alone (in B/Yamanashi/166/1998 recombinant viruses) [11,29]. However, D198E was observed for the last time in 2001 in the Yamagata lineage, and it is possible that this substitution does not confer a RI phenotype any longer in the contemporary B/Phuket/3073/2013 viral background. The D198N susceptibility results are in agreement with recent surveillance data, where a B/Wyoming/07/2017 (B/Yamagata lineage) variant had an RI phenotype to peramivir, was susceptible to zanamivir and had a borderline RI phenotype to oseltamivir (5.8-fold increase of $\mathrm{IC}_{50}$ value compared to WT) [15]. In addition, our N294S recombinant exhibited an RI phenotype to zanamivir, whereas this mutant was previously described as susceptible [29]. Thus, the susceptibility phenotype for some NA substitutions may vary depending on the viral genetic backgrounds. In addition, the viral evolution may introduce NA substitutions whose accumulation over time may 
interfere with the susceptibility results when associated with NAI-resistance mutations. For example, the role of the D198N NA substitution on RI/HRI phenotypes could not be confirmed in the B/Singapore/GP702/2015 virus [30]. Thus, based on these observations, genotypic characterization of clinical isolates may not be sufficient to determine the susceptibility of a specific variant, and phenotypic testing could still be required.

The impact of NA mutations on the in vitro viral fitness of our recombinants viruses was further assessed. We did not observe a significant difference between viral titers of the mutants compared to the WT, except at the $72 \mathrm{~h}$ p.i. time-point where reduced titers were observed for the R152K mutants $(p<0.01$ vs WT) (Figure 1). Of note, viral replication of our recombinant H136N mutant was not impaired while this substitution altered the fitness of B/Laos/0406/2016 virus [28]. The difference in the lineage between the two strains (Yamagata for our strain vs Victoria for B/Laos/0406/2016) could be responsible for such discrepancy.

Besides in vitro properties, we also seek to characterize our recombinant viruses using an in vivo system. Although the mouse model does not constitute the gold standard for studying the replication and transmission of influenza B viruses to the same extent as ferrets, it may provide a technical and cost-favorable option for evaluating some experimental parameters, such as weight loss, mortality rates, and lung viral titers. However, mice are not naturally susceptible to IBV infections, and multiple lung-to-lung passages resulting in numerous HA/M/NP/PA mutations are usually needed to adapt a specific IBV virus strain to that animal species [31]. Interestingly, the $B /$ Phuket/3073/2013 vaccine strain and its recombinant virus were found to be lethal in BALB/c mice without prior adaptation, when using a high viral inoculum (i.e., $5 \times 10^{6} \mathrm{PFUs}$ ). Indeed, no mortality could be observed when we used a lower inoculum of $5 \times 10^{5}$ PFUs which is in agreement with a previous study [32]. Noteworthily, the characterization of the viral fitness in our model does not take into account the potential effect of mutations that could emerge during the mouse adaptation process [31]. As the original B/Phuket/3073/2013 vaccine strain was passaged in embryonated eggs, we identified in its HA protein the N196D egg-adapted substitution that could potentially increase viral pathogenicity in mice through adaptation to a $\alpha 2,3$ sialic acid receptor. A similar role was previously attributed for the S190R A(H1N1) egg-adapted HA mutation [33,34].

In our study, all mice infected with H136N, I222T and N294S recombinant viruses died, contrasting with the R152K recombinant virus that killed only 33\% of the mice (Figure 2B). Thus, weight loss and mortality data demonstrated that in vivo viral fitness was not altered in multi-resistant NA mutants (except for R152K). Although the in vitro replicative capacity of R152K variant was not impaired, the in vivo-reduced viral fitness for this variant could be explained by the fact that the R152 is a catalytic residue contrasting to the remaining mutations, which are rather framework substitutions (Table 1). In addition, we showed that, in contrast to the other mutant NAs, the recombinant R152K NA enzyme had lower activity when compared to that of the WT NA (Table 1). A previous study demonstrated that the influenza NA activity has a more important role in vivo than in MDCK cell-culture context due to the presence of a mucin/surfactant-rich environment in the respiratory tract [35]. The ability of an influenza virus to disseminate through the mucus barrier is dependent on its NA activity that prevents viruses from being trapped [36].

\section{Conclusions}

In conclusion, our approach using a contemporary and pathogenic IBV recombinant strain confirms that most NA substitutions associated with NAI resistance in previous strains still confer a cross-resistance phenotype in the B/Phuket/3073/2013 background. However, we noted some discrepancies with regard to the D198E/N variants, highlighting the potential variability of NAI susceptibility results between different strains. More interestingly, we demonstrated that some NAI-resistant IBV strains could retain viral fitness in vitro and in a mouse model. The impact of these mutations on viral transmission remains to be clarified by further experiments in ferrets. These findings warrant continuous surveillance programs and reinforce the need to develop novel antiviral strategies. 
Author Contributions: C.F., Y.A. and G.B. conceived and designed the experiments; C.F., L.C. and M.-C.V performed the experiments; C.F., Y.A. and G.B. analyzed the data; C.F. and Y.A. wrote the paper.

Funding: This work was supported by a Canadian Institutes of Health Research (CIHR) foundation grant to GB (grant No. 229733) for a research program on the pathogenesis, treatment, and prevention of respiratory and herpes viruses.

Conflicts of Interest: The authors declare no conflict of interest.

\section{References}

1. Shaw, M.W.; Xu, X.; Li, Y.; Normand, S.; Ueki, R.T.; Kunimoto, G.Y.; Hall, H.; Klimov, A.; Cox, N.J.; Subbarao, K. Reappearance and global spread of variants of influenza B/victoria/2/87 lineage viruses in the 2000-2001 and 2001-2002 seasons. Virology 2002, 303, 1-8. [CrossRef] [PubMed]

2. Ran, Z.; Shen, H.; Lang, Y.; Kolb, E.A.; Turan, N.; Zhu, L.; Ma, J.; Bawa, B.; Liu, Q.; Liu, H.; et al. Domestic pigs are susceptible to infection with influenza B viruses. J. Virol. 2015, 89, 4818-4826. [CrossRef]

3. Molinari, N.A.; Ortega-Sanchez, I.R.; Messonnier, M.L.; Thompson, W.W.; Wortley, P.M.; Weintraub, E.; Bridges, C.B. The annual impact of seasonal influenza in the us: Measuring disease burden and costs. Vaccine 2007, 25, 5086-5096. [CrossRef] [PubMed]

4. Burnham, A.J.; Baranovich, T.; Govorkova, E.A. Neuraminidase inhibitors for influenza B virus infection: Efficacy and resistance. Antivir. Res. 2013, 100, 520-534. [CrossRef]

5. Su, S.; Chaves, S.S.; Perez, A.; D’Mello, T.; Kirley, P.D.; Yousey-Hindes, K.; Farley, M.M.; Harris, M.; Sharangpani, R.; Lynfield, R.; et al. Comparing clinical characteristics between hospitalized adults with laboratory-confirmed influenza A and B virus infection. Clin. Infect. Dis. 2014, 59, 252-255. [CrossRef] [PubMed]

6. Huang, S.S.; Banner, D.; Paquette, S.G.; Leon, A.J.; Kelvin, A.A.; Kelvin, D.J. Pathogenic influenza B virus in the ferret model establishes lower respiratory tract infection. J. Gen. Virol. 2014, 95, 2127-2139. [CrossRef] [PubMed]

7. Adlhoch, C.; Snacken, R.; Melidou, A.; Ionescu, S.; Penttinen, P. The European Influenza Surveillance Network. Dominant influenza A(H3N2) and B/Yamagata virus circulation in eu/eea, 2016/17 and 2017/18 seasons, respectively. Euro Surveill. 2018, 23. [CrossRef]

8. Colman, P.M.; Hoyne, P.A.; Lawrence, M.C. Sequence and structure alignment of paramyxovirus hemagglutinin-neuraminidase with influenza virus neuraminidase. J. Virol. 1993, 67, 2972-2980.

9. Food and Drugs Administration (FDA). Influenza (Flu) Antiviral Drugs and Related Information. 2018. Available online: https:/ / www.fda.gov/Drugs/DrugSafety/InformationbyDrugClass/ucm100228.htm (accessed on 30 November 2018).

10. Gubareva, L.V.; Besselaar, T.G.; Daniels, R.S.; Fry, A.; Gregory, V.; Huang, W.; Hurt, A.C.; Jorquera, P.A.; Lackenby, A.; Leang, S.K.; et al. Global update on the susceptibility of human influenza viruses to neuraminidase inhibitors, 2015-2016. Antivir. Res. 2017, 146, 12-20. [CrossRef] [PubMed]

11. Hurt, A.C.; Iannello, P.; Jachno, K.; Komadina, N.; Hampson, A.W.; Barr, I.G.; McKimm-Breschkin, J.L. Neuraminidase inhibitor-resistant and -sensitive influenza B viruses isolated from an untreated human patient. Antimicrob. Agents Chemother. 2006, 50, 1872-1874. [CrossRef]

12. Hurt, A.C.; Besselaar, T.G.; Daniels, R.S.; Ermetal, B.; Fry, A.; Gubareva, L.; Huang, W.; Lackenby, A.; Lee, R.T.; Lo, J.; et al. Global update on the susceptibility of human influenza viruses to neuraminidase inhibitors, 2014-015. Antivir. Res. 2016, 132, 178-185. [CrossRef] [PubMed]

13. Samson, M.; Pizzorno, A.; Abed, Y.; Boivin, G. Influenza virus resistance to neuraminidase inhibitors. Antivir. Res. 2013, 98, 174-185. [CrossRef] [PubMed]

14. Tu, V.; Abed, Y.; Barbeau, X.; Carbonneau, J.; Fage, C.; Lague, P.; Boivin, G. The I427T neuraminidase (NA) substitution, located outside the NA active site of an influenza A(H1N1)pdm09 variant with reduced susceptibility to NA inhibitors, alters NA properties and impairs viral fitness. Antivir. Res. 2017, 137, 6-13. [CrossRef] [PubMed]

15. Lackenby, A.; Besselaar, T.G.; Daniels, R.S.; Fry, A.; Gregory, V.; Gubareva, L.V.; Huang, W.; Hurt, A.C.; Leang, S.K.; Lee, R.T.C.; et al. Global update on the susceptibility of human influenza viruses to neuraminidase inhibitors and status of novel antivirals, 2016-2017. Antivir. Res. 2018, 157, 38-46. [CrossRef] [PubMed] 
16. World Health Ogranization (WHO). Laboratory Methodologies for Testing the Antiviral Susceptibility of Influenza Viruses: Neuraminidase Inhibitor (NAI). 2018. Available online: http:/ / www.who.int/influenza/ gisrs_laboratory/antiviral_susceptibility/nai_overview/en/ (accessed on 30 November 2018).

17. Hatakeyama, S.; Sakai-Tagawa, Y.; Kiso, M.; Goto, H.; Kawakami, C.; Mitamura, K.; Sugaya, N.; Suzuki, Y.; Kawaoka, Y. Enhanced expression of an alpha2,6-linked sialic acid on MDCK cells improves isolation of human influenza viruses and evaluation of their sensitivity to a neuraminidase inhibitor. J. Clin. Microbiol. 2005, 43, 4139-4146. [CrossRef] [PubMed]

18. Zhou, B.; Lin, X.; Wang, W.; Halpin, R.A.; Bera, J.; Stockwell, T.B.; Barr, I.G.; Wentworth, D.E. Universal influenza B virus genomic amplification facilitates sequencing, diagnostics, and reverse genetics. J. Clin. Microbiol. 2014, 52, 1330-1337. [CrossRef]

19. Hoffmann, E.; Neumann, G.; Kawaoka, Y.; Hobom, G.; Webster, R.G. A DNA transfection system for generation of influenza A virus from eight plasmids. Proc. Natl.Acad. Sci. USA 2000, 97, 6108-6113. [CrossRef]

20. Fage, C.; Tu, V.; Carbonneau, J.; Abed, Y.; Boivin, G. Peramivir susceptibilities of recombinant influenza A and B variants selected with various neuraminidase inhibitors. Antivir. Ther. 2017, 22, 711-716. [CrossRef]

21. Hayden, F.G.; Sugaya, N.; Hirotsu, N.; Lee, N.; de Jong, M.D.; Hurt, A.C.; Ishida, T.; Sekino, H.; Yamada, K.; Portsmouth, S.; et al. Baloxavir marboxil for uncomplicated influenza in adults and adolescents. N. Engl. J. Med. 2018, 379, 913-923. [CrossRef]

22. Omoto, S.; Speranzini, V.; Hashimoto, T.; Noshi, T.; Yamaguchi, H.; Kawai, M.; Kawaguchi, K.; Uehara, T.; Shishido, T.; Naito, A.; et al. Characterization of influenza virus variants induced by treatment with the endonuclease inhibitor baloxavir marboxil. Sci. Rep. 2018, 8, 9633. [CrossRef]

23. World Health Ogranization (WHO). Recommended Composition of Influenza Virus Vaccines for Use in the 2015-2016 Northern Hemisphere Influenza Season. 2015. Available online: http:/ / www.who.int/influenza/ vaccines/virus/recommendations/2015_16_north/en/ (accessed on 30 November 2018).

24. Public Health Agency of Canada (PHAC). Fluwatch Report: 22 July 2018 to 25 August 2018 (Weeks 30-34). 2018. Available online: https://www.canada.ca/en/public-health/services/publications/diseasesconditions / fluwatch/2017-2018/week30-34-july-22-august-25-2018.html (accessed on 30 November 2018).

25. Gubareva, L.V.; Matrosovich, M.N.; Brenner, M.K.; Bethell, R.C.; Webster, R.G. Evidence for zanamivir resistance in an immunocompromised child infected with influenza B virus. J. Infect. Dis. 1998, 178, 1257-1262. [CrossRef] [PubMed]

26. Ison, M.G.; Gubareva, L.V.; Atmar, R.L.; Treanor, J.; Hayden, F.G. Recovery of drug-resistant influenza virus from immunocompromised patients: A case series. J. Infect. Dis. 2006, 193, 760-764. [CrossRef] [PubMed]

27. Carr, S.; Ilyushina, N.A.; Franks, J.; Adderson, E.E.; Caniza, M.; Govorkova, E.A.; Webster, R.G. Oseltamivir-resistant influenza A and B viruses pre- and postantiviral therapy in children and young adults with cancer. Pediatr. Infect. Dis. J. 2011, 30, 284-288. [CrossRef] [PubMed]

28. Baranovich, T.; Vongphrachanh, P.; Ketmayoon, P.; Sisouk, T.; Chomlasack, K.; Khanthamaly, V.; Nguyen, H.T.; Mishin, V.P.; Marjuki, H.; Barnes, J.R.; et al. Antiviral drug-resistant influenza B viruses carrying H134N substitution in neuraminidase, laos, february 2016. Emerg. Infect. Dis. 2017, 23, 686-690. [CrossRef] [PubMed]

29. Burnham, A.J.; Baranovich, T.; Marathe, B.M.; Armstrong, J.; Webster, R.G.; Govorkova, E.A. Fitness costs for influenza B viruses carrying neuraminidase inhibitor-resistant substitutions: Underscoring the importance of E119A and H274Y. Antimicrob. Agents Chemother. 2014, 58, 2718-2730. [CrossRef] [PubMed]

30. Farrukee, R.; Zarebski, A.E.; McCaw, J.M.; Bloom, J.D.; Reading, P.C.; Hurt, A.C. Characterization of influenza B virus variants with reduced neuraminidase inhibitor susceptibility. Antimicrob. Agents Chemother. 2018, 62, e01081-18. [CrossRef] [PubMed]

31. Kim, E.H.; Park, S.J.; Kwon, H.I.; Kim, S.M.; Kim, Y.I.; Song, M.S.; Choi, E.J.; Pascua, P.N.; Choi, Y.K. Mouse adaptation of influenza B virus increases replication in the upper respiratory tract and results in droplet transmissibility in ferrets. Sci. Rep. 2015, 5, 15940. [CrossRef]

32. Pascua, P.N.Q.; Mostafa, H.H.; Marathe, B.M.; Vogel, P.; Russell, C.J.; Webby, R.J.; Govorkova, E.A. Pathogenicity and peramivir efficacy in immunocompromised murine models of influenza B virus infection. Sci. Rep. 2017, 7, 7345. [CrossRef]

33. Lugovtsev, V.Y.; Vodeiko, G.M.; Strupczewski, C.M.; Ye, Z.; Levandowski, R.A. Generation of the influenza B viruses with improved growth phenotype by substitution of specific amino acids of hemagglutinin. Virology 2007, 365, 315-323. [CrossRef] 
34. Chen, Y.; Bai, T.; Zhu, W.; Gao, R.; Deng, Z.; Shi, Y.; Zou, S.; Huang, Y.; Li, X.; Li, F.; et al. The S190R mutation in the hemagglutinin protein of pandemic H1N1 2009 influenza virus increased its pathogenicity in mice. Sci. China Life Sci. 2018, 61, 836-843. [CrossRef]

35. Cohen, M.; Zhang, X.Q.; Senaati, H.P.; Chen, H.W.; Varki, N.M.; Schooley, R.T.; Gagneux, P. Influenza a penetrates host mucus by cleaving sialic acids with neuraminidase. Virol. J. 2013, 10, 321. [CrossRef] [PubMed]

36. Yang, X.; Steukers, L.; Forier, K.; Xiong, R.; Braeckmans, K.; Van Reeth, K.; Nauwynck, H. A beneficiary role for neuraminidase in influenza virus penetration through the respiratory mucus. PLoS ONE 2014, 9, e110026. [CrossRef] [PubMed]

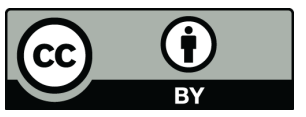

(C) 2018 by the authors. Licensee MDPI, Basel, Switzerland. This article is an open access article distributed under the terms and conditions of the Creative Commons Attribution (CC BY) license (http:/ / creativecommons.org/licenses/by/4.0/). 